\title{
BRCA1 NP_009225.1:p.E23Vfs
}

National Cancer Institute

\section{Source}

National Cancer Institute. BRCA1 NP 009225.1:p.E23Vfs. NCI Thesaurus. Code C131474.

A change in the amino acid sequence of breast cancer type 1 susceptibility protein where a deletion mutation results in the replacement of the glutamic acid at position 23 with a valine immediately followed by a stop codon. 